\title{
The impact of the Type D Personality pattern on prehospital delay in patients suffering from acute myocardial infarction
}

\author{
Youyang Zhang ${ }^{1}$, Shihao Wu ${ }^{2}$, Jiangqi Pan ${ }^{3}$, Sophia Hoschar ${ }^{4,5}$, Zhen Wang ${ }^{6}$, Rongxiang Tu ${ }^{1}$, \\ Karl-Heinz Ladwig ${ }^{7 \#}$, Wenlin $\mathrm{Ma}^{1,2 \#}$ \\ ${ }^{1}$ Department of Cardiology, Shanghai Tongji Hospital, Tongji University School of Medicine, Shanghai, China; ${ }^{2}$ Department of Geriatrics, Shanghai \\ Tongji Hospital, Tongji University School of Medicine, Shanghai, China; ${ }^{3}$ Department of Cardiology, Gongli Hospital, Navy Military Medical \\ University, Shanghai, China; ${ }^{4}$ Institute of Epidemiology, Mental Health Research Unit, Helmholtz Zentrum München, German Research Center for \\ Environmental Health (HMGU), Neuherberg, Germany; ${ }^{5}$ Department of Psychosomatic Medicine and Psychotherapy, Medical Center-University \\ of Freiburg, Faculty of Medicine, Freiburg, Germany; ${ }^{6}$ Department of General Practice, Jiangning Hospital, Nanjing Medical University, Nanjing, \\ China; ${ }^{7}$ Department of Psychosomatic Medicine and Psychotherapy, University Hospital Rechts der Isar, Technische Univerität Munich (TUM), \\ Munich, Germany \\ Contributions: (I) Conception and design: Y Zhang, KH Ladwig; (II) Administrative support: W Ma; (III) Provision of study materials or patients: W \\ Ma; (IV) Collection and assembly of data: J Pan, S Hoschar, Z Wang, R Tu; (V) Data analysis and interpretation: Y Zhang, S Wu; (VI) Manuscript \\ writing: All authors; (VII) Final approval of manuscript: All authors. \\ \#These authors shared senior authorship. \\ Correspondence to: Prof. Wenlin Ma, MD. Department of Geriatrics, Tongji Hospital of Tongji University, 389 Xincun Rd., Putuo District, Shanghai, \\ China. Email: mawenlin@tongji.edu.cn.
}

Background: The Type D Personality (TDP) has been specifically linked to acute myocardial infarction (AMI). However, the impact on prehospital delay of AMI patients is unclear. The aim of this study was to assess the relationship between TDP and pre-hospital delay time (PHT) in a Chinese population.

Methods: A total of 256 AMI patients (47 women and 209 men) were taken from the Multicenter Delay in Patients Experiencing AMI in Shanghai (MEDEA FAR-EAST) study. Sociodemographic and psychobehavioral characteristics were assessed by bedside interviews and questionnaires. TDP was evaluated according to the Type D Personality Scale (DS14) subdivided in social inhibition (SI) and negative affectivity (NA). Based on a significant interaction analysis of TDP and sex on PHT, all analyses were stratified by sex.

Results: PHT of female patients with TDP were substantially shorter compared to non-TDP female patients (108 vs. $281 \mathrm{~min}, \mathrm{P}=0.029)$. In male patients, no effect of TDT on PHT was found. Spearman correlation analysis suggests that NA was negatively correlated with PHT ( $r=-0.358, \mathrm{P}=0.014)$. Further age-adjusted logistic regression analyses showed that female patients with TDP were generally less likely to prehospital delay compared with non-TDP patients $(\mathrm{OR}=0.28 ; 95 \% \mathrm{CI}, 0.08-0.98)$ and had a lower risk of PHT >360 minutes (OR =0.10; 95\% CI, 0.01-0.91). However, statistical significance disappeared after adjustment for psychological factors (anxiety, depression, suboptimal wellbeing, cardiac denial and stress event).

Conclusions: TDP is associated with less prehospital delay in female patients during AMI-an effect which may be particularly mediated by NA.

Keywords: Type D Personality (TDP); acute myocardial infarction (AMI); prehospital delay; decision time

Submitted Apr 01, 2020. Accepted for publication Aug 03, 2020.

doi: $10.21037 /$ jtd-20-1546

View this article at: http://dx.doi.org/10.21037/jtd-20-1546 


\section{Introduction}

Short- and long-term outcomes in patients with acute myocardial infarction (AMI) are closely related to the time of reperfusion (1-4). The risk of mortality is likely to increase by $7.5 \%$ for each 30 -minute delay in treatment (2). Implementation of organizational strategies achieved significant door-to-treatment time reductions but with very little improvement of patients' overall mortality (5). Of note, the prehospital delay time (PHT) accounts for $60 \%$ to $70 \%$ of the total delay time which is mainly driven by the patient's decision time (6). Moreover, patients with prehospital delay are also more likely to experience delay in hospital treatment (7). The key to reducing the total delay time may be effectively shorten PHT.

Identification of factors influencing prehospital delays may help to shorten PHT. Previous studies on influencing factors of PHT focused on sociodemographic factors, context conditions at symptom onset, and clinical symptoms of AMI (8-11) while the importance of psychological factors contributing to PHT is increasingly acknowledged only recently.

Type D Personality (TDP) is characterized by negative affectivity (NA, the tendency to experience negative emotions) and social inhibition (SI, the tendency to inhibit the expression of emotions) (12). Studies have shown that TDP may be a psychological risk factor associated with the incidence and progression of coronary heart disease $(13,14)$ and thus be an independent predictor of cardiac and noncardiac mortality (15). However, the impact of TDP on prehospital delay has not been investigated so far. Considering that this personality trait may affect individual performance and decision making in the face of an AMI, we aimed to analyze the impact of TDP on prehospital delay of Chinese AMI patients. We present the following article in accordance with the TRIPOD reporting checklist (available at http://dx. doi. org/10. 21037/jtd-20-1546).

\section{Methods}

The data of this investigation stem from the MEDEA FAREAST Study, a multi-center cross-sectional study designed to elucidate in-depth barriers contributing to delay in Chinese AMI-patients. The detailed design of the MEDEA FAR-EAST Study has been described previously (16). The study was conducted in accordance with the Declaration of Helsinki (as revised in 2013). The study was approved by the Ethics Commission of Tongji-University affiliated
Tongji-Hospital (KYSB-2016-74) and informed consent was taken from all the patients. This approval is applicable for all participating centers (Tongji-Hospital, Yangpu-Hospital, Tenth-Hospital and 455 Hospital).

\section{Setting and sample}

From April 2016 to January 2017, a total of 296 patients were routinely screened in the acute coronary care units of four cardiology departments in Shanghai (Tongji-Hospital, Tenth-Hospital, Yangpu-Hospital and 455 People's Hospital). The major inclusion criterion was hospitalization with an AMI, no restrictions were made regarding age and sex. AMI was confirmed by typical symptoms at onset, and elevated cardiac biomarkers (troponin I or troponin $\mathrm{T}$ ) as well as a corresponding ECG-diagnosis. The exclusion criteria were out-of-hospital cardiac arrest, cognitive impairment and language barriers. After excluding patients without valid data of the Type D Personality Scale (DS14) assessment, a total of 256 patients were included in our study.

All patients underwent a three-part evaluation. First, the trained study personnel conducted a bedside interview. Thereafter, a self-administered questionnaire was handled to the patients for self-assessment. Finally, basic epidemiological and medical information were collected from the hospital's patient charts. All patients received information about the purpose and procedures of the study, and signed a declaration of consent.

All study personnel received training prior to their participation and were closely monitored during the data acquisition process, when implementing Standardized Operation Procedures (SOP) and tracking any missing or incoherent answers by phone in order to avoid reporting bias.

\section{Measures}

\section{Prehospital delay}

PHT (prehospital delay) was the primary outcome defined as the time interval between symptom-onset and arrival at the hospital door, measured in minutes. Asking patients to recall the time point when the acute symptoms began remained a challenge. Therefore, the onset-time was triangulated by trained personnel in the interview (17), using events from the patient's daily routine to help them establish the chronology of symptom-onset to minimize recall bias. 
Based on the triangulation process, we were able to calculate the PHT. PHT did not approximate a normal distribution after logarithmic transformation; therefore, several categorical outcomes were built. In replication of outcomes used in previous studies, two dichotomous outcomes were considered: using cut-offs of 2 and $6 \mathrm{~h}$. Furthermore, PHT was categorized into 5-classifications: $\leq 60,61-120,121-240,241-360,>360 \mathrm{~min}$.

DT (patient's decision delay) was also an important outcome defined as the elapsed time from symptom onset to the decision to seek medical care or call EMS. We then calculated the DT and categorized the times as $<60$ and $\geq$ 60 minutes during analysis.

\section{TDP}

TDP was evaluated by the DS14 scale, including two subscales: SI and NA subscale. Both subscales included 7 items ranging from 0 (false) to 4 (true), with item 1 and 3 scored in reverse. Both SI score and NA score $\geq 10$ points indicated D-type personality tendency (18). A Chinese version of the DS14 instrument was used that has been validated in a Chinese patient sample with coronary heart disease. The NA and SI scales were internally consistent (Cronbach's alpha $=0.89 / 0.81)(19)$.

\section{Psychological measures}

Anxiety was assessed with the Generalized Anxiety Disorder scale (GAD-7) composed of 7 items, rated on a 4-point Likert scale from not present to very high, leading to an overall score ranging from $0-21$. GAD-7 score $\geq 10$ points highlights anxious patients (20).

Depression was assessed using the major depression inventory (MDI), which generates DSM-IV and ICD10 diagnoses by the patients' self-reported symptoms. Depression is defined as at least five symptoms present in the MDI scale, of which at least one must be a 'core' symptom (core symptoms including lack of energy, depressed mood and lack of interests). A scoring of 4-5 (i.e., most of the time, all the time) for the 'core' symptom categories and a scoring from 3-5 (i.e., more than half of the time, most of the time and all the time) for the remaining items was mandatory to define depression (21).

Subjective wellbeing was evaluated through the WHO5 , as one of the most widely used questionnaires for measuring happiness, energy, motivation and interest in daily life. It contains five items on a 6-point scale ranging from 0 to 25 . Multiplication by 4 results in a total score of 0 to 100 . WHO- 5 score $\leq 50$ indicates suboptimal well- being (22).

Denial regarding cardiac illness was assessed by the Cardiac Denial of Impact Scale (CDIS). The overall score ranges from 8 to 40 . A score of $\geq 25$ indicates cardiac denial (23). Patients were asked to rate the severity of the symptomatic pain from $0-10$ points according, with 10 being the most painful. Subjective risk perception was selfassessed $(1=$ very low, $2=$ rather low, $3=$ medium, $4=$ high, $5=$ very high), 1-3 indicated insufficient risk perception. Fear of death and stress event were asked to answer "yes" or "no".

\section{Data analysis}

The differences in sociodemographic and clinical characteristics of patients with TDP compared to non-TDP were calculated using the Chi-square test. Differences in age, PHT and decision time were assessed using the non-parametric Wilcoxon test. The correlation between PHT, DT and SI score, NA score was explored using spearman correlation analysis. A logistic regression model was applied to assess the association between TDP and PHT (>120 and >360 $\mathrm{min}$ ), DT (>60 min). Multinomial logistic regression was used for the 5 -classification model (PHT: $\leq 60,61-120,121-240,241-360,>360 \mathrm{~min})$. The relative risk for longer delay is presented as odds ratio (OR) with 95\% confidence interval ( $95 \% \mathrm{CI})$.

SPSS 23.0 was used for all statistical analysis, $\mathrm{P}<0.05$ was considered statistically significant. The analysis and description in this paper follow the STROBE guidelines for cross-sectional studies (24).

\section{Results}

The present study population consisted of $256 \mathrm{AMI}$ patients, including 47 (18.4\%) women and 209 (81.6\%) men. Age ranged from 32 to 90 years (mean age $=62.5$, SD $=13.1 ; \mathrm{IQR}=14$ ). In the total sample, the median PHT was 150 minutes. A total of 139 (54.3\%) AMI patients reported a prehospital delay of more than 120 minutes; among them $\mathrm{n}=78(30.5 \%)$ patients with more than 360 minutes prehospital delay.

\section{Prevalence and distribution of TDP in patients with AMI}

The overall prevalence of TDP was $24.6 \%$ (men: $24.4 \%$; women: $25.5 \%$ ). As shown in Table 1, patients with TDP were more likely to be younger $(63 \pm 16$ vs. $59 \pm 11, \mathrm{P}=0.03)$, living alone $(\mathrm{P}=0.006)$. Concerning clinical conditions and somatic risk factors, patients with TDP experienced 
Table 1 Sociodemographic and clinical characteristics of the study population stratified by TDP ( $\mathrm{n}=63$ ) and non-TDP ( $\mathrm{n}=193$ )

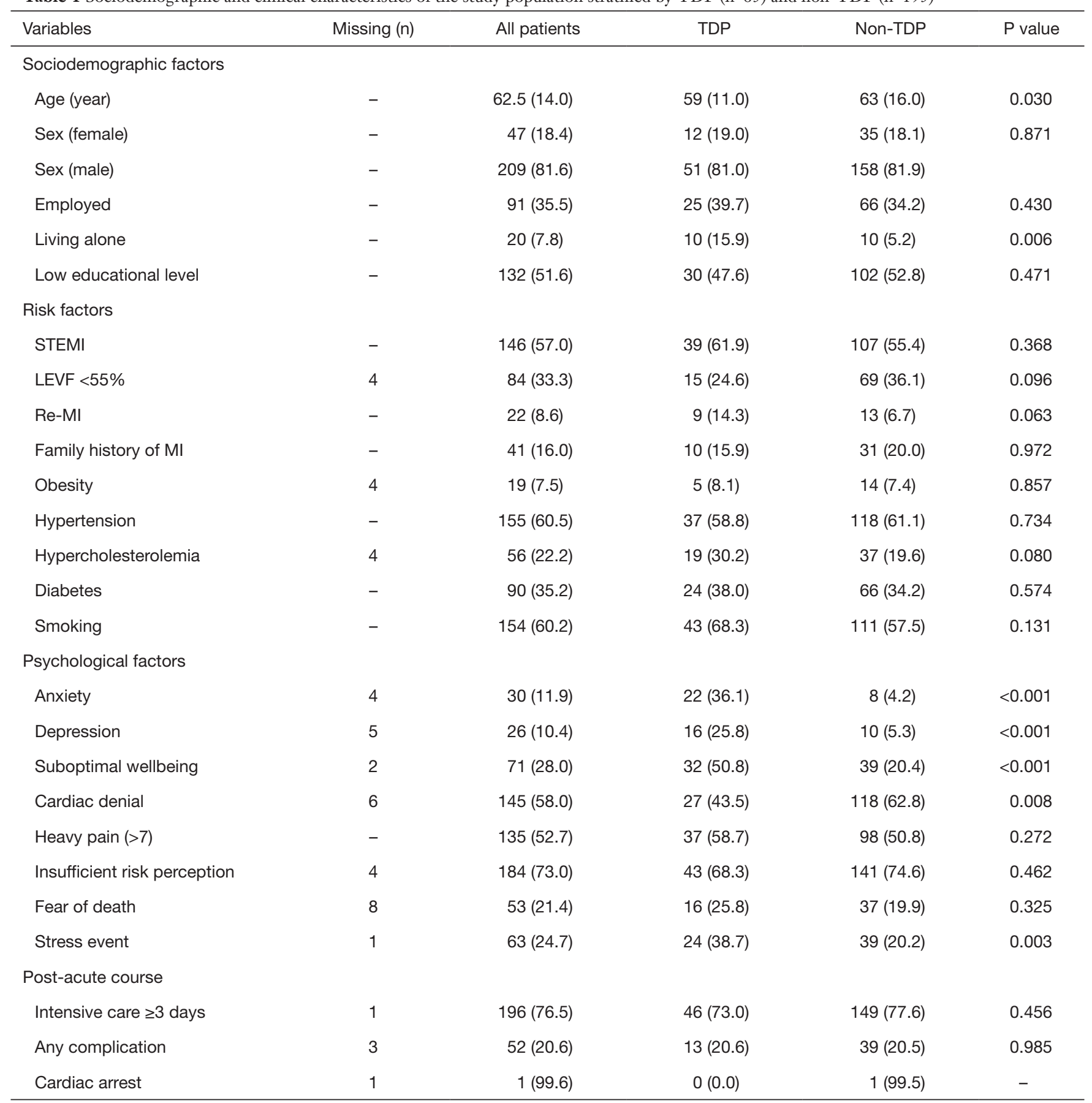

Data were shown as $\mathrm{n}(\%)$, except age which was shown as median (IQR). TDP, Type D Personality; STEMI, ST-segment elevation myocardial infarction; LEVF, left ventricular ejection fraction; MI, myocardial infarction. Low educational level is defined as junior high school and below.

a tendency to have a higher prevalence of recurrent MI $(\mathrm{P}=0.063)$ and hypercholesterolemia $(\mathrm{P}=0.080)$. However, no significant differences were found for gender, educational level, and employment status.
Furthermore, when considering the medical history of the post-acute infarction phase (intensive care, complications and cardiac arrest), we did not observe any significant differences (Table 1). However, in female 

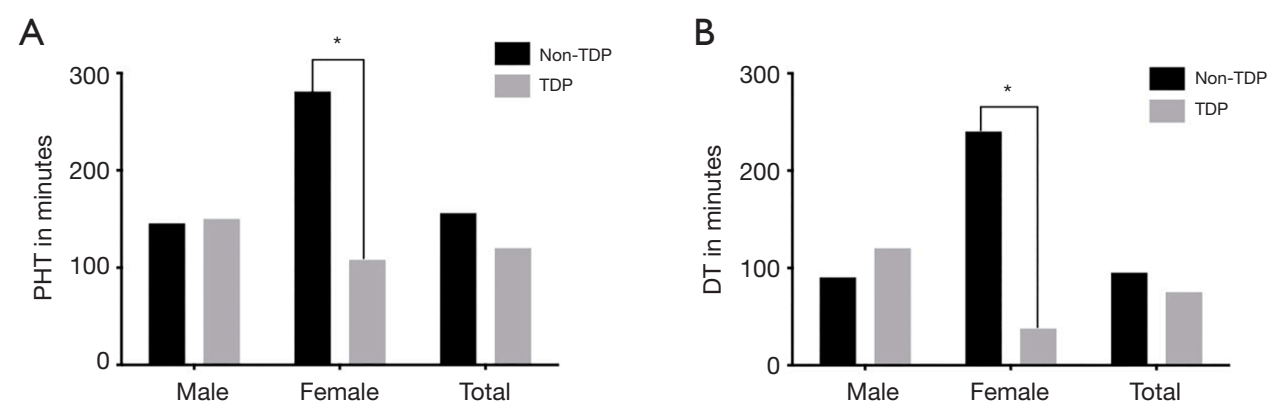

Figure 1 Non-parametric Wilcoxon test for comparing PHT and DT (in min) for all patients with and without TDP and stratified for women and men. The punctuation '*' represented $\mathrm{P}<0.05$. (A) The difference of PHT in men: non-TDP: 145.5 (391.3) vs. TDP: 150.0 (455.0), $\mathrm{P}=0.832$. For women: non-TDP: 281.0 (978.0) vs. TDP: 108.0 (173.8), $\mathrm{P}=0.029$. For the total population: non-TDP: 156.0 (469.0) vs. TDP: 120.0 (379.0), $\mathrm{P}=0.492$. (B) The difference of DT for men: non-TDP:90.0 (343.5); TDP: 120.0 (390.0), P=0.931. For women: nonTDP: 240.0 (970.0) vs. TDP: 37.5 (127.5), $\mathrm{P}=0.032$. For the total population: non-TDP: 95.0 (410.0); TDP: 75.0 (340.0), P=0.433. Data were shown as median (IQR). PHT, pre-hospital delay time; TDP, Type D Personality.

Table 2 The correlation between two traits of TDP and PHT, DT in male and female AMI patients

\begin{tabular}{|c|c|c|c|c|c|c|c|c|}
\hline \multirow{2}{*}{ Variables } & \multicolumn{4}{|c|}{ Men } & \multicolumn{4}{|c|}{ Women } \\
\hline & $r$ & $P$ & $r$ & $P$ & $r$ & $P$ & $r$ & $P$ \\
\hline PHT & -0.039 & 0.577 & -0.070 & 0.314 & -0.150 & 0.326 & -0.354 & 0.014 \\
\hline DT & -0.024 & 0.737 & -0.091 & 0.192 & -0.152 & 0.308 & -0.351 & 0.016 \\
\hline
\end{tabular}

TDP, Type D Personality; PHT, pre-hospital delay time; AMI, acute myocardial infarction.

patients, we found that patients with TDP were more likely to have complications and intensive care $\geq 3$ days, although not statistically significant (Table S1).

\section{Psychological characteristics of patients with TDP during the 6 months prior to $A M I$}

As displayed in Table 1, not surprisingly, patients with TDP were more likely to report suboptimal wellbeing $(\mathrm{P}<0.0001)$, depression $(\mathrm{P}<0.0001)$, anxiety $(\mathrm{P}<0.0001)$ and stress events $(\mathrm{P}=0.003)$, but were less likely to be cardiac deniers $(43.5 \%$ vs. $62.8 \%, \mathrm{P}=0.008)$.

\section{Gender differences in $\mathrm{PHT}$ and $\mathrm{DT}$}

An interaction analysis of TDP and sex on prehospital delay revealed a significant synergistic effect of TDP and female sex on PHT $(\mathrm{P}=0.047)$ which prompted us to stratify all further analyses by sex. As can be seen in Figure 1, we found that the PHT and DT of female patients with TDP were substantially shorter compared to non-TDP female patients (108 vs. 281 and 38 vs. $240 \mathrm{~min}$, respectively). In male patients, there was no association of TDT on PHT or DT at all.

In order to elucidate the pronounced sex differences in delay time associated with TDP, we performed a sensitivity analysis on psychological differences between TDP men and women but failed to confirm differences for anxiety and depression. Women also did not perceive stronger pain at $\mathrm{AMI}$ onset and did not report more insufficient risk perception than their male counterparts (data not shown). However, the proportion of women living alone was higher (41.7\% vs. $9.8 \%, \mathrm{P}=0.016)$.

\section{Correlation between DS14 score and PHT, DT}

The results of the Spearman correlation analyses of TDP subscales with delay parameters are shown in Table 2. In women, we found that the NA score was negatively correlated with PHT and DT ( $\mathrm{r}=-0.358, \mathrm{P}=0.014$ and $\mathrm{r}=-0.351, \mathrm{P}=0.016$ respectively). There was also a weak 


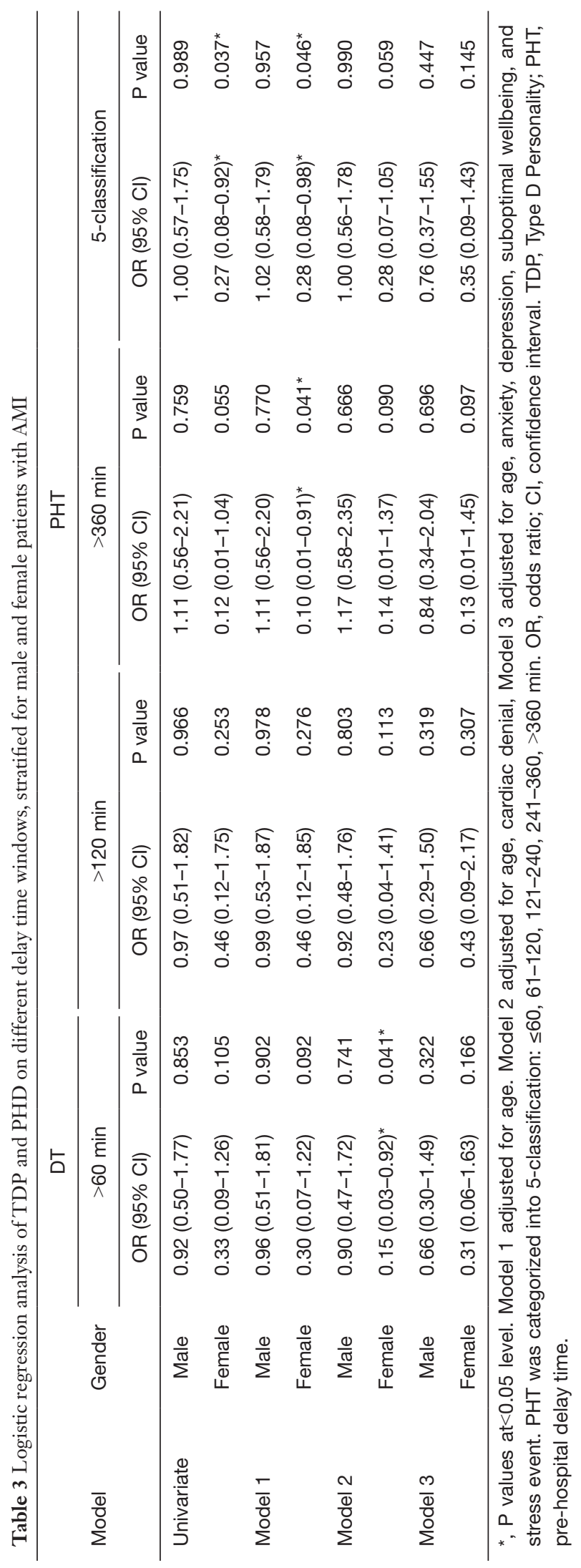

negative correlation trend in men. No correlation was found for the SI score. This may indicate that NA (but not SI) within the TDP pattern exerts a dominant influence on prehospital delay.

\section{Impact of TDP on prehospital delay and patient's decision delay}

We investigated the impact of TDP in different time windows on prehospital DT and PHT. As can be seen in Table 3, the univariate logistic regression analysis revealed that the TDP was associated with arriving in an shorter PHT classification in women (5-classification: $\leq 60,61-120$, 121-240, 241-360 and $>360 \mathrm{~min}$ ). After adjusting for age (model 1), we found that female patients with TDP were less likely to delay $>360 \mathrm{~min}(\mathrm{OR}=0.10 ; 95 \% \mathrm{CI}$, $0.01-0.91$ ), and the association remained significant in the 5-classification scheme (OR $=0.28 ; 95 \%$ CI, 0.08-0.98). However, after further adjusting for cardiac denial (model 2), anxiety, depression, suboptimal wellbeing, and stress event (model 3), the significance was lost. The impact of TDP on DT was statistically significant after adjusting for age and cardiac denial (OR $=0.15$; 95\% CI, 0.03-0.92). Female patients with TDP experienced shorter decision times (Table 3). Again, no statistically significant effects were found for male patients.

\section{Discussion}

To our knowledge, this is the first comprehensive evaluation of the impact of TDP on the prehospital delay of a homogeneous study population of Chinese AMI patients in the immediate wake after an acute life-threatening coronary heart syndrome. Driven by a significant interaction of TDP and sex on PHT, we performed stratified analyses for both sexes disclosing a remarkable sex dimorphism. The major finding of the present investigation is to show that women suffering from TDP experience significant and clinically meaningful shorter PHT and DT than their non-affected female counterparts. While the median PHT for women with TDP was $108 \mathrm{~min}$, the corresponding delay in nonTDP female patients summed up to a critical augmented median PHT of $281 \mathrm{~min}$. In consequence, adjusted regression analyses confirmed this finding by showing a highly inverse and significant OR for the $>360 \mathrm{~min}$ and the 5-classification time window. Most interesting, such an association was not found in the male subgroup.

The Type D concept compiles two distinct components: 
NA and SI (25). Of note, the Spearman correlation analysis of NA and SI components in this investigation revealed that the NA score-again exclusively in female patientswas significantly correlated with the two outcome factors (PHT,DT) indicating that the "favorable" effect of TDP on decision and delay time in women operated solely through NA and not through SI. Neither, we found a similar correlation for the SI component nor within the male study group.

To the best of our knowledge, this is the second study reporting the impact of TDP on prehospital decision time. Moreover, our study had a bigger sample size and simultaneously analyzed the effect of TDP on PHT. The previously mentioned report (26) was a small retrospective study conducted in Spain, including 16 women and 86 men. They investigated the effects of some psychological factors, including TDP, on patient's decision delay, and found that TDP may be a risk factor for more decision delay after the cardiac event, which seems to be contrary to our research results. It is worth noting that in this Spanish study, before the regression model included serious consequences, TDP did not correlate significantly with decision delay (all samples). At this level, it is completely consistent with our research. This may indicate that both sex and serious consequences mediated the effect of TDP on decision delay, and produced different effects.

Schlyter et al. focused on personality factors and applied the five-factor model (FFM) including neuroticism, extraversion, openness, agreeableness, and conscientiousness. Their findings suggested no significant association on PHT (27). Albarqouni et al. showed that fear of death in the face of an acute MI promoted early arrival in coronary care emergency departments (28) and Fang et al. evidenced that suffering from generalized anxiety disorder (GAD) was also preventive- even after adjustment for fear of death and depression (29). Depression has been associated with increased prehospital delays in patients with acute coronary syndrome (30), AMI (31), and heart failure (32). On the other hand, evidence suggests that high scores of denial rating facilitate clinically significant PHTs (33-35). Counterintuitively, O'Carroll et al. found that AMI patients who waited over 4 hours prior to seeking medical help scored lower on neuroticism, denial and health locus of control (chance) (33).

Moreover, the impact of TDP on PHT and DT is not entirely surprising: Michal et al. studied a representative population based sample of $n=2,495$ subjects in Europe with a prevalence of $33.1 \%$ suffering from TDP and evidenced that even after comprehensive adjustment, Type D still remained associated with increased help seeking behavior and utilization of health care (36). TDP in the present investigation clusters significantly with depression, anxiety and suboptimal wellbeing confirming a bulk of studies which showed a strong association of TDP with various forms of NA such as anxiety $(37,38)$ and high self-perceived psychosocial stress $(38,39)$. Furthermore, TDP subjects were more likely to live alone, to experience stronger pain during the acute phase of the heart attack and to employ significantly less often denial as coping style-all factors which are likely to increase awareness of the malignant context of the AMI.

The finding of a substantial sex dimorphism concerning the impact of TDP on PHT and DT came unexpected. Currently, we do not have a firm interpretation of these findings. However, apparently for male patients, the adverse conditions compiled in TDP do not operate as "cues for action" and are still not effective in provoking help seeking behavior and thus are less able to launch adequate actions. For women, the opposite is true: the data clearly show that women respond highly sensitive on the threatening interoceptive stimuli.

Although TDP was not associated with acute complications and adverse events during the early days of hospitalization in the general population, there was a trend for fewer adverse events (intensive care $\geq 3$ days, $\mathrm{P}=0.071$; any complication, $\mathrm{P}=0.166$ ) among female patients, which is consistent with their fewer PHT. And previous studies have shown that TDP was an independent predictor of cardiovascular outcomes $(13,14)$ despite conflicting evidence from other studies who failed to replicate the mortality risk of TDP $(40,41)$. Unfortunately, this study did not follow-up evaluation of long-term adverse events and mortality. The favorable delay time associated with TDP in female AMI patients, as evidenced in the present investigation, is likely to be one of the important factors which should be taken into consideration when discussing the mortality risk of TDP.

The main strength of our study is the comprehensive assessment of patients' psychological conditions based on multi-center data and highly standardized assessment methods during hospital care. Yet, there are study limitations that are worth considering. First, the small size, especially in female patients compromises advanced subgroup analyses, therefore, replications of these results in larger datasets would be desirable. Second, the PHT was assessed retrospectively giving room for a recall bias 
although the onset-time was triangulated by trained and routinely supervised personnel. In addition, up to onethird of all patients with an AMI die without reaching a hospital (42), selection bias could have resulted from only accounting survivors.

\section{Conclusions}

The present study shows TDP may increase awareness of the malignant context of the AMI in female (not in male) patients leading to less time spent on making decisions and thus may contribute to waste less PHT.

\section{Acknowledgements}

The authors would like to thank all MEDEA FAR-EAST interviewers for their work and Dr. Wu Heng for providing us with advice on the final instrument. We also thank all the patients who participated in the study.

Funding: This work was supported by the Science and Technology Committee Foundation of Shanghai, PR China $(16411965500,16511102802)$ to Prof. Ma Wenlin and by the German Heart Foundation to Prof. Karl-Heinz Ladwig. The funding was used to cover printing fees and to pay the study personnel.

\section{Footnote}

Reporting Checklist: The authors have completed the TRIPOD reporting checklist. Available at http://dx.doi. org/10.21037/jtd-20-1546

Data Sharing Statement: Available at http://dx.doi. org/10.21037/jtd-20-1546

Peer Review File: Available at http://dx. doi. org/10. 21037/ jtd-20-1546

Conflicts of Interest: All authors have completed the ICMJE uniform disclosure form (available at http://dx. doi. org/10.21037/jtd-20-1546). The authors have no conflicts of interest to declare.

Ethical Statement: The authors are accountable for all aspects of the work in ensuring that questions related to the accuracy or integrity of any part of the work are appropriately investigated and resolved. The study was conducted in accordance with the Declaration of Helsinki (as revised in 2013). The study was approved by the Ethics Commission of Tongii-University affiliated Tongji-Hospital (KYSB-2016-74) and informed consent was taken from all the patients. This approval is applicable for all participating centers (Tongii-Hospital, Yangpu-Hospital, Tenth-Hospital and 455 Hospital).

Open Access Statement: This is an Open Access article distributed in accordance with the Creative Commons Attribution-NonCommercial-NoDerivs 4.0 International License (CC BY-NC-ND 4.0), which permits the noncommercial replication and distribution of the article with the strict proviso that no changes or edits are made and the original work is properly cited (including links to both the formal publication through the relevant DOI and the license). See: https://creativecommons.org/licenses/by-nc-nd/4.0/.

\section{References}

1. Koul S, Andell P, Martinsson A, et al. Delay from first medical contact to primary PCI and all-cause mortality: a nationwide study of patients with ST-elevation myocardial infarction. J Am Heart Assoc 2014;3:e000486.

2. De Luca G, Suryapranata H, Ottervanger JP, et al. Time delay to treatment and mortality in primary angioplasty for acute myocardial infarction: every minute of delay counts. Circulation 2004;109:1223-5.

3. Guerchicoff A, Brener SJ, Maehara A, et al. Impact of delay to reperfusion on reperfusion success, infarct size, and clinical outcomes in patients with ST-segment elevation myocardial infarction: the INFUSE-AMI Trial (INFUSE-Anterior Myocardial Infarction). JACC Cardiovasc Interv 2014;7:733-40.

4. $\mathrm{Ng} \mathrm{S}$, Ottervanger JP, van 't Hof AW, et al. Impact of ischemic time on post-infarction left ventricular function in ST-elevation myocardial infarction treated with primary percutaneous coronary intervention. Int J Cardiol 2013;165:523-7.

5. Bagai A, Dangas GD, Stone GW, et al. Reperfusion strategies in acute coronary syndromes. Circ Res 2014;114:1918-28.

6. Sasson C, Rogers MA, Dahl J, et al. Predictors of survival from out-of-hospital cardiac arrest: a systematic review and meta-analysis. Circ Cardiovasc Qual Outcomes 2010;3:63-81.

7. Ting HH, Bradley EH, Wang Y, et al. Delay in presentation and reperfusion therapy in ST-elevation myocardial infarction. Am J Med 2008;121:316-23. 
8. Sheifer SE, Rathore SS, Gersh BJ, et al. Time to presentation with acute myocardial infarction in the elderly: associations with race, sex, and socioeconomic characteristics. Circulation 2000;102:1651-6.

9. Wang X, Hsu LL. Treatment-seeking delays in patients with acute myocardial infarction and use of the emergency medical service. J Int Med Res 2013;41:231-8.

10. Kendall H, Marley A, Patel JV, et al. Hospital delay in South Asian patients with acute ST-elevation myocardial infarction in the UK. Eur J Prev Cardiol 2013;20:737-42.

11. Saczynski JS, Yarzebski J, Lessard D, et al. Trends in prehospital delay in patients with acute myocardial infarction (from the Worcester Heart Attack Study). Am J Cardiol 2008;102:1589-94.

12. Denollet J, Sys SU, Brutsaert DL. Personality and mortality after myocardial infarction. Psychosom Med 1995;57:582-91.

13. Pedersen SS, Lemos PA, van Vooren PR, et al. Type D personality predicts death or myocardial infarction after bare metal stent or sirolimus-eluting stent implantation: a Rapamycin-Eluting Stent Evaluated at Rotterdam Cardiology Hospital (RESEARCH) registry substudy. J Am Coll Cardiol 2004;44:997-1001.

14. Leu HB, Yin WH, Tseng WK, et al. Impact of type D personality on clinical outcomes in Asian patients with stable coronary artery disease. J Formos Med Assoc 2019;118:721-9.

15. Denollet J, Sys SU, Stroobant N, et al. Personality as independent predictor of long-term mortality in patients with coronary heart disease. Lancet 1996;347:417-21.

16. Hoschar S, Pan J, Wang Z, et al. The MEDEA FAREAST Study: Conceptual framework, methods and first findings of a multicenter cross-sectional observational study. BMC Emerg Med 2019;19:31.

17. Moser DK, McKinley S, Dracup K, et al. Gender differences in reasons patients delay in seeking treatment for acute myocardial infarction symptoms. Patient Educ Couns 2005;56:45-54.

18. Emons WH, Meijer RR, Denollet J. Negative affectivity and social inhibition in cardiovascular disease: evaluating type-D personality and its assessment using item response theory. J Psychosom Res 2007;63:27-39.

19. Yu DS, Thompson DR, Yu CM, et al. Validating the Type $\mathrm{D}$ personality construct in Chinese patients with coronary heart disease. J Psychosom Res 2010;69:111-8.

20. Spitzer RL, Kroenke K, Williams JB, et al. A brief measure for assessing generalized anxiety disorder: the GAD-7. Arch Intern Med 2006;166:1092-7.
21. Bech P, Rasmussen NA, Olsen LR, et al. The sensitivity and specificity of the Major Depression Inventory, using the Present State Examination as the index of diagnostic validity. J Affect Disord 2001;66:159-64.

22. Topp CW, Ostergaard SD, Sondergaard S, et al. The WHO-5 Well-Being Index: a systematic review of the literature. Psychother Psychosom 2015;84:167-76.

23. Fowers BJ. The cardiac denial of impact scale: a brief, self-report research measure. J Psychosom Res 1992;36:469-75.

24. von Elm E, Altman DG, Egger M, et al. The Strengthening the Reporting of Observational Studies in Epidemiology (STROBE) Statement: guidelines for reporting observational studies. Int J Surg 2014;12:1495-9.

25. Denollet J. DS14: standard assessment of negative affectivity, social inhibition, and Type D personality. Psychosom Med 2005;67:89-97.

26. Arrebola-Moreno M, Petrova D, Garrido D, et al. Psychosocial markers of pre-hospital decision delay and psychological distress in acute coronary syndrome patients. Br J Health Psychol 2020;25:305-23.

27. Schlyter M, Andre-Petersson L, Engstrom G, et al. The impact of personality factors on delay in seeking treatment of acute myocardial infarction. BMC Cardiovasc Disord 2011;11:21.

28. Albarqouni L, von Eisenhart Rothe A, Ronel J, et al. Frequency and covariates of fear of death during myocardial infarction and its impact on prehospital delay: findings from the multicentre MEDEA Study. Clin Res Cardiol 2016;105:135-44.

29. Fang XY, Spieler D, Albarqouni L, et al. Impact of generalized anxiety disorder (GAD) on prehospital delay of acute myocardial infarction patients. Findings from the multicenter MEDEA study. Clin Res Cardiol 2018;107:471-8.

30. Wong CK, Tang EW, Herbison P, et al. Pre-existent depression in the 2 weeks before an acute coronary syndrome can be associated with delayed presentation of the heart attack. QJM 2008;101:137-44.

31. Bunde J, Martin R. Depression and prehospital delay in the context of myocardial infarction. Psychosom Med 2006;68:51-7.

32. Johansson P, Nieuwenhuis M, Lesman-Leegte I, et al. Depression and the delay between symptom onset and hospitalization in heart failure patients. Eur J Heart Fail 2011;13:214-9.

33. O'Carroll RE, Smith KB, Grubb NR, et al. Psychological factors associated with delay in attending hospital following 
a myocardial infarction. J Psychosom Res 2001;51:611-4.

34. Stenström U, Nilsson AK, Stridh C, et al. Denial in patients with a first-time myocardial infarction: relations to pre-hospital delay and attendance to a cardiac rehabilitation programme. Eur J Cardiovasc Prev Rehabil 2005;12:568-71.

35. Fang XY, Albarqouni L, von Eisenhart Rothe AF, et al. Is denial a maladaptive coping mechanism which prolongs pre-hospital delay in patients with ST-segment elevation myocardial infarction? J Psychosom Res 2016;91:68-74.

36. Michal M, Wiltink J, Grande G, et al. Type D personality is independently associated with major psychosocial stressors and increased health care utilization in the general population. J Affect Disord 2011;134:396-403.

37. Kupper N, Denollet J. Type D personality is associated with social anxiety in the general population. Int $\mathrm{J}$ Behav Med 2014;21:496-505.

38. Smith MA, Riccalton VC, Kelly-Hughes DH, et al. The relationship between Type D personality and physical

Cite this article as: Zhang Y, Wu S, Pan J, Hoschar S, Wang Z, Tu R, Ladwig KH, Ma W. The impact of the Type D Personality pattern on prehospital delay in patients suffering from acute myocardial infarction. J Thorac Dis 2020;12(9):46804689. doi: $10.21037 /$ jtd-20-1546 health complaints is mediated by perceived stress and anxiety but not diurnal cortisol secretion. Stress 2018;21:229-36.

39. Howard S, Hughes BM. Construct, concurrent and discriminant validity of Type $\mathrm{D}$ personality in the general population: associations with anxiety, depression, stress and cardiac output. Psychol Health 2012;27:242-58.

40. Condén E, Rosenblad A, Wagner P, et al. Is type D personality an independent risk factor for recurrent myocardial infarction or all-cause mortality in postacute myocardial infarction patients? Eur J Prev Cardiol 2017;24:522-33.

41. Coyne JC, Jaarsma T, Luttik ML, et al. Lack of prognostic value of type $\mathrm{D}$ personality for mortality in a large sample of heart failure patients. Psychosom Med 2011;73:557-62.

42. Tydén P. Myocardial infarction in an urban population - Studies on patterns of disease in terms of time, place and person. Available online: https://lup.lub.lu.se/search/ publication/6e01c420-4947-45a6-b433-fdb589a29c5b 
Supplementary

Table S1 The medical history of the post-acute infarction phase in female TDP $(n=12)$ and non-TDP $(n=35)$ patients

\begin{tabular}{lccc}
\hline Variables & TDP & Non-TDP & P value \\
\hline Intensive care $\geq 3$ days & $1(8.3)$ & $14(40.0)$ & 0.071 \\
Any complication & $0(0.0)$ & $7(20.0)$ & 0.166 \\
Cardiac arrest & $0(0.0)$ & $0(0.0)$ & - \\
\hline
\end{tabular}

TDP, Type D Personality. 